\title{
Effect of Rootstock, Soil and Foliar Nutrition in Some Vegetative Characteristics Growth of Local Lemon Seedlings Citrus Limon L.
}

\author{
Sahar Hussein Tkhyel ${ }^{\mathrm{a}} \quad$ Raad Taha Mohammed Ali Balaket $^{\mathrm{b}}$ \\ ${ }^{a, b}$ AlForat Alawsat Technical University, Technical College Musayib \\ Saharh917@gmail.com_ RaadBalakett@yahoo.com
}

Submission date:- 29/7/2018 Acceptance date:- 5/9/2018 Publication date:- 15/1/2019

Keywords: Lemon, Soil nutrient, Foliar nutrient, Rootstock.

\begin{abstract}
An experiment was conducted in the wooden canopy affiliated to the Technical College AL-Musaib during the period from the beginning of March until the end of December, 2017 on local lemon citron seedlings aged seven months and grafted on the two seed roots Sour orange and Sweet lemon to study the effect spray and soil fertilizers feeding of organic TARASOIL CALCIO with concentration $(0,50,100) \mathrm{ml}^{-\mathrm{L}^{-1}}$ and sprays of DECSON with concentration of $(0,10,20) \mathrm{ml}^{-\mathrm{L}^{-1}}$ to study the characteristics of vegetative, The soil fertilization in $11 / 4 / 2017$, $11 / 5 / 2017,11 / 9 / 2017$ and 11/10/2017, foliar spray in $7 / \Sigma / 2017,7 / 5 / 2017,7 / 9 / 2017$ and 7/10/2017, the experiment was applied by Completely Randomized Design(C.R.D)as factorial experiment $(2 \times 3 \times 3)$ with three replicates and three seedling and 162 seedlings as a total group. The results were analyzed using statistical analysis program(Excel). The averages were compared according to the least significant difference(L.S.D)at probability level of (0.05), The type of rootstock have a significant effect on vegetative, The rootstock of sweet lemon increased plant height, leaves number, leaf area, while rootstock sour orange increased in stem, scion. Stalk of sweet lemon $+100 \mathrm{ml} . \mathrm{L}^{-1}$ TARASOIL CALCIO had a suppressive effect in plant height, leaves number while rootstock sour orange suppressive in stock stem, scion, leaf area, Stalk of sweet lemon $+20 \mathrm{ml} . \mathrm{L}^{-1}$ DECSON had a suppressive effect in plant height, leaf number, leaf area while Sour crease stalk a suppressive in main stem, scion. sweet lemon stalk+100ml.L-1 TARASOIL CALCIO+20 ml.L ${ }^{-1}$ DECSON had a suppressed in plant height, scion, leaf number, leaf area, while stalk the orange had a suppressive effect in stock stem.
\end{abstract}

\section{تأثير الأصل و التغذية الأرضية والورقية في بعض صفات النمو الخضري لثتلات الليمون Citrus Limon L. حامض المحلي}

رعد طه محمد علي بلاكت

جامعة الفرات الأوسط التقنية ، الكلية التقنية، المسيب

RaadBalakett@yahoo.com

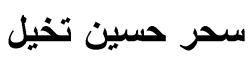

Saharh917@gmail.com

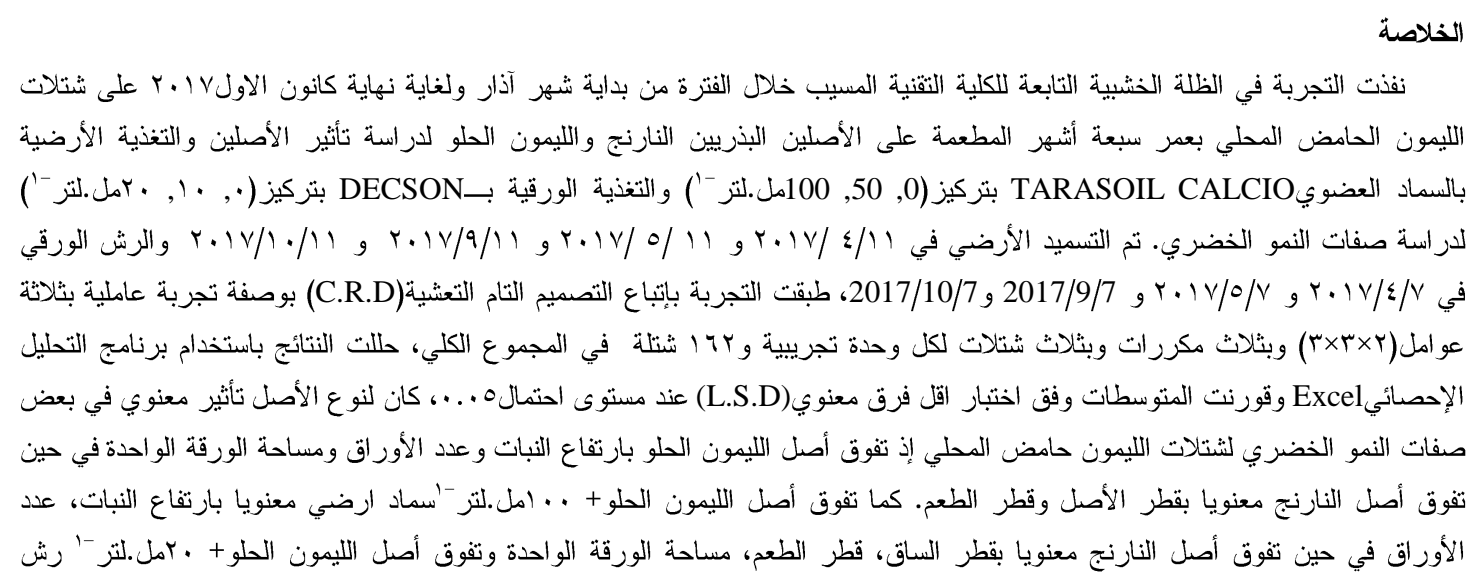

Journal of University of Babylon for Pure and Applied Sciences (JUBAS) by University of Babylon is licened under a Creative Commons Attribution 4.0 International License. 2018. 


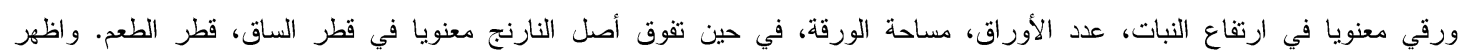

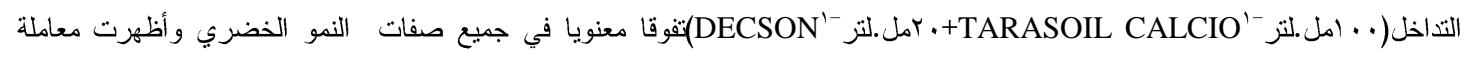

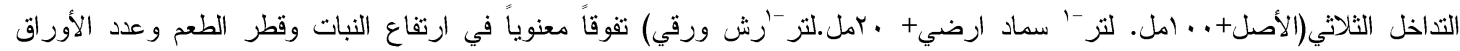
ومساحة الورقة في حين تفوق أصل النارنج معنوياً بقطر الساق. لأنس. الكلمات الا الة:الليمون حامض المحلي, التغذية الأرضية، التغذية الورقية، الأصل.

الحمضيات من نباتات الفاكهة المستديمة والتي تعود إلى العائلة السذبية Rutaceae وتضم العديد من الأجناس أهمها الجنس Citrus والذي

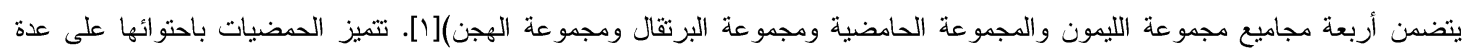

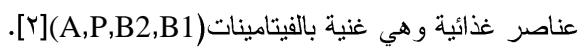
تتميز ثمار الليمون حامض المحلي بكونها غنية بالأملاح المعدنية اللازمة لبناء جسم الإنسان مثل البوتأستابيوم، المغنسيوم, الكالسيوم, الحديد,

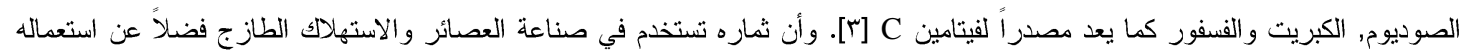
كمطيبات لكثير من الأطعمة كما إن له الأثر الفعال في علاج العديد من الأمر اض[ـــ، إن الطريقة الثائعة في إكثار الأصناف التجارية للحمضيات

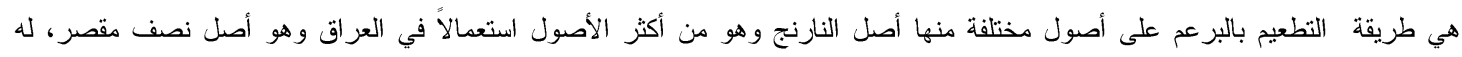

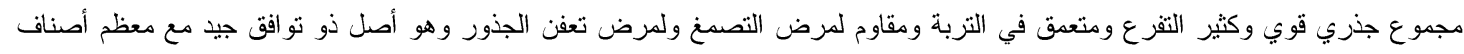

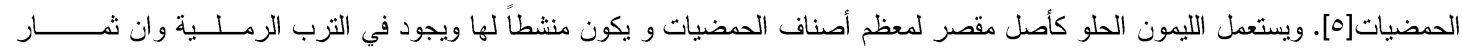

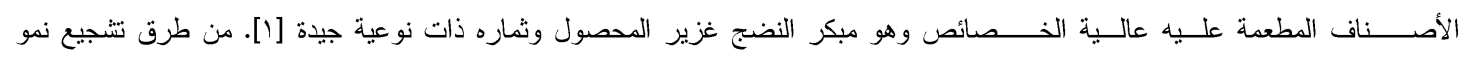

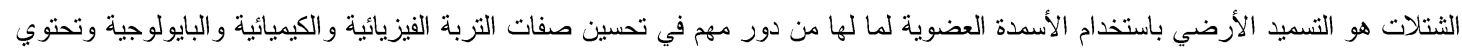

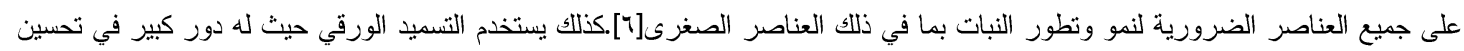

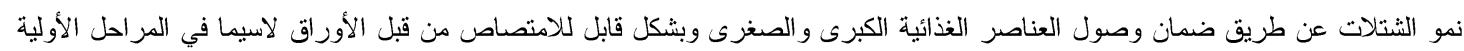

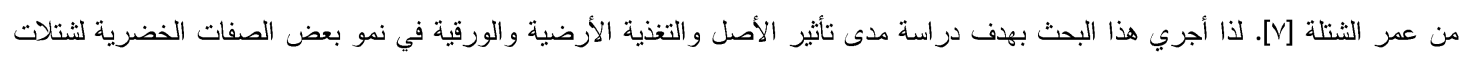

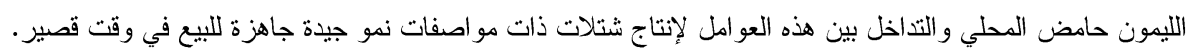

المواد وطر ائق العمل: نفذ البحث في الظلة الخشبية في الكلية التقنية المسيب. تم اختيار 162 شتلة متجانسة قدر الإمكان أصلاً ثانياً، وبعمر 7 أثهر حيث ملئت

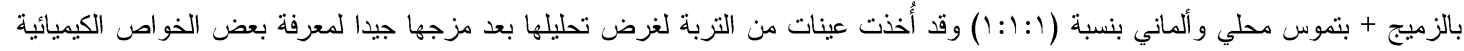

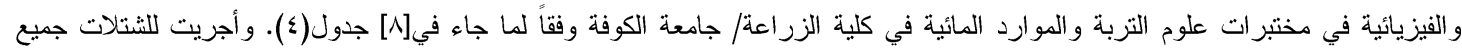

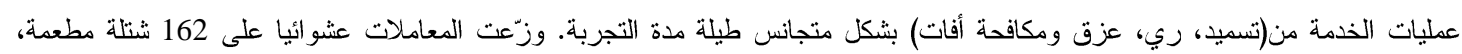

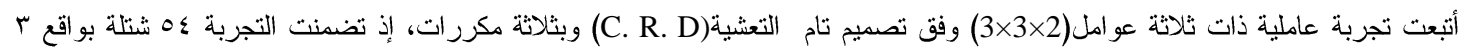
شتلات لكل وحدة تجريبية في المكرر الواحد.

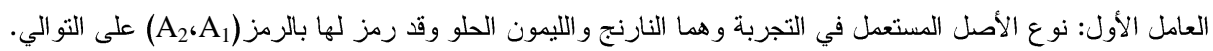

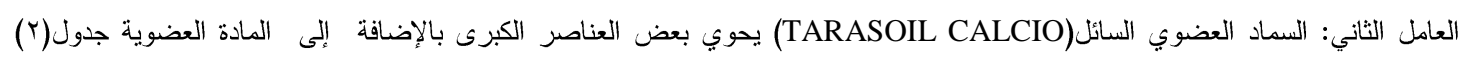

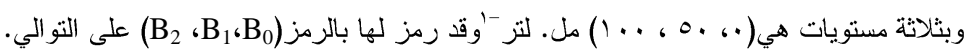

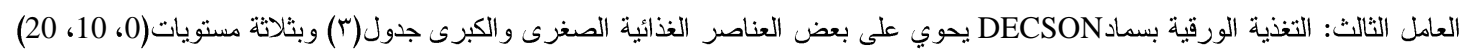
مل. لتر - وقد رمز لها بالرمز

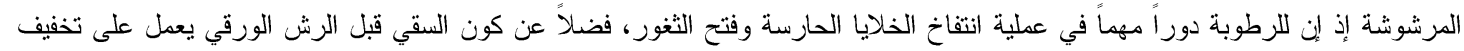

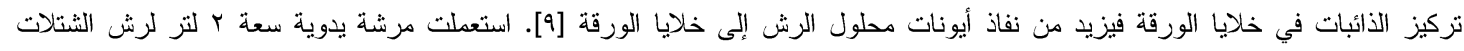

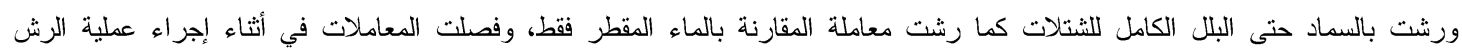

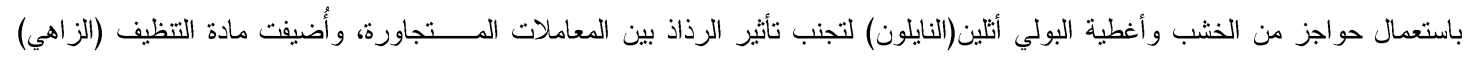

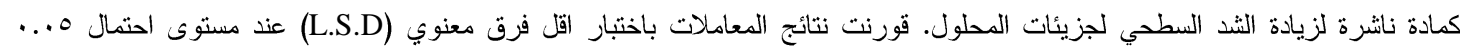

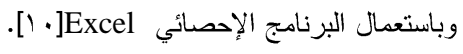




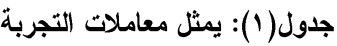

\begin{tabular}{|c|c|c|c|}
\hline رمز أصل الليمون الحلو & رمز أصل النارنج & التركيز مل.لتر -1 & ت \\
\hline A2 B0 C0 & A1 B0 C0 & $\mathbf{0 , 0}$ & 1 \\
\hline A2 B1 C0 & A1 B1 C0 & 50,0 & $r$ \\
\hline A2 B2 C0 & A1 B2 C0 & 100,0 & $r$ \\
\hline A2 B0 C1 & A1 Bo C1 & $\mathbf{0 , 1 0}$ & $\varepsilon$ \\
\hline A2 B1 C1 & A1 B1 C1 & 50,10 & 0 \\
\hline A2 B2 C1 & A1 B2 C1 & 100,10 & 7 \\
\hline A2 B0 C2 & A1 B0 C2 & $\mathbf{0 , 2 0}$ & $v$ \\
\hline A2 B1 C2 & A1 B1 C2 & 50,20 & $\wedge$ \\
\hline A2 B2 C2 & A1 B2 C2 & 100,20 & 9 \\
\hline
\end{tabular}

جدول (ץ) التركيب الكيميائي للسماد الأرضي (TARASOIL CALCIO)

\begin{tabular}{|c|c|}
\hline التركيب & النسبة المئوية \\
\hline $\mathbf{N}$ & $\%$ \\
\hline Nitric nitrogen & $\%$ \\
\hline $\mathrm{CaO}$ & $\% 14$. \\
\hline Fulvic acid & $\%$ r $\varepsilon_{\text {. }}$ \\
\hline
\end{tabular}

جدول (r) التركيب الكيميائي للسماد الورقي(DECSON)

\begin{tabular}{|c|c|c|c|c|c|c|c|c|c|}
\hline $\mathbf{T i}$ & Mo & $\mathbf{C a}$ & $\mathrm{Zn}$ & $\mathrm{Cu}$ & B & Mg & Mn & $\mathrm{Fe}$ & التركيب \\
\hline$\% \ldots$ & $\% \ldots$ & \%.r.ro & $\%$. r & $\% \ldots \wedge$ & $\%$.. \&० & $\%$. . r & $\%$. . & $\%$. . & النسبة \\
\hline
\end{tabular}

جدول(؛) بعض الصفات الفيزيائية و الكيميائية للتربة المستخدمة بالتجربة

\begin{tabular}{|c|c|c|}
\hline وحدة القياس & قيمة التحليل & نوع التحليل \\
\hline غم. كفم -1 & $9 .$. & الرمل \\
\hline غم. كفم -1 & Y.V & الطين \\
\hline غم. كفم -1- & V.r & الغرين \\
\hline- & تربة رملية & نسجة التربة \\
\hline- & V.r & درجة التفاعل PH \\
\hline ديسي سيمنز . م-1 & t.rV & التوصيل الكهربائي Ec \\
\hline غم. كغم 1- & 10.1 & المادة العضوية O.M \\
\hline ملغم. لتر -1 & r.or & النتروجين \\
\hline ملغم .لتر 1- & r.\&A & الفسفور \\
\hline ملفم. لتر -1 & YAI & البوتاسيوم \\
\hline ملفم. لتر -1 & $\ldots V 4$ & الحديد \\
\hline ملغم. لتر 1- & ru.l. & الكالسيوم \\
\hline ملفم. لتر-1 & 17.4 & الصوديوم \\
\hline
\end{tabular}

الصفات المدروسة

1-متوسط ارتفاع الثتلةة(سم):

قيس باستعمال شريط القياس المتري وأجري قياس الارتفاع من سطح التربة وحتى القمة النامية ولكل وحدة تجرييية.

r- متوسط قطر الساق الرئيسي (ملم):

قيس قطر الساق الرئيسي اللشتلات باستعمال القدمة Vernia وحسب المتوسط لكل وحدة تجريية. 
ب- متوسط قطر الطعم (ملم): قيس قطر الطعم للشتلات باستعمال القدمة Vernia وحسب المتوسط لكل وحدة تجريبية.

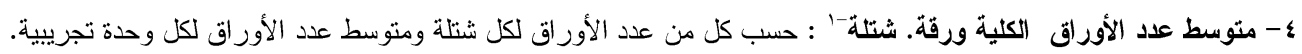

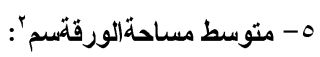
قيست مساحة الورقة بواسطة جهاز PLANIMETER في مختبرات قسم التربة و الموارد المائية في المعهد التقني المسيب/ جامعة الفرات الأوسط التقنية وقد أخذت تسع أور اق كاملة من أماكن مختلفة من النبات الواحد وقيست المساحة الورقية لها وحساب المتوسط لكل شتلة ثم اجري حساب المتوسط لكل وحدة تجريبية.

النتائج و المناقشة:

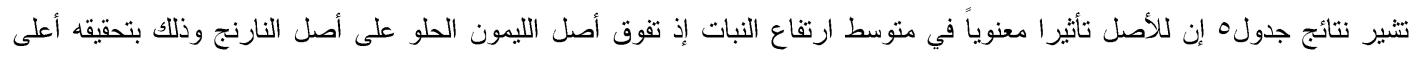

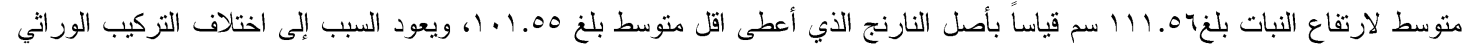

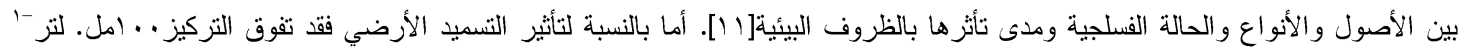

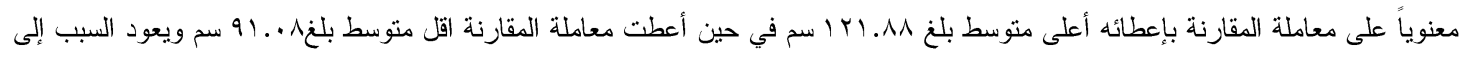

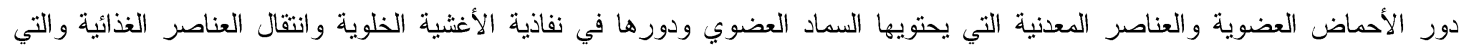

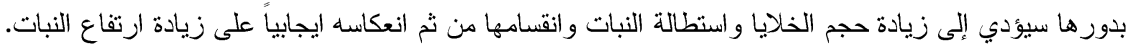

\begin{tabular}{|c|c|c|c|c|c|}
\hline \multirow[t]{2}{*}{ A.B } & \multicolumn{3}{|c|}{ 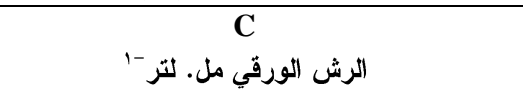 } & \multirow{2}{*}{ 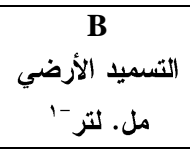 } & \multirow[t]{2}{*}{$\begin{array}{c}\text { الأصل } \\
\end{array}$} \\
\hline & $\mathbf{C}_{2}$ & $\mathbf{C}_{1}$ & $\mathrm{C}_{0}$ & & \\
\hline $1 \wedge .97$ & $91 . .$. & AN.VY & Av.17 & $\mathbf{B}_{\mathbf{0}}$ & \multirow[t]{3}{*}{ A1 النارنج A1 } \\
\hline 99.89 & 1.r.s. & $91 . .0$ & 97.90 & $\mathbf{B}_{1}$ & \\
\hline 117.10 & Irr.Ir & $110 . \varepsilon r$ & $11 \ldots r$ & $\mathbf{B}_{2}$ & \\
\hline $9 r .41$ & $1.4 .9 \varepsilon$ & 97 & $1 . . v 1$ & $\mathbf{B}_{0}$ & \multirow{3}{*}{$\begin{array}{c}\text { الليمون الحلو } \\
\text { A2 }\end{array}$} \\
\hline 111.97 & $111 . \wedge 4$ & 114.9 & $11 . .14$ & $\mathbf{B}_{1}$ & \\
\hline \multirow[t]{2}{*}{ Irv.or } & $1 \% \varepsilon . r 4$ & Irv.0 & Ir..Ar & $\mathbf{B}_{2}$ & \\
\hline & $11 \mathrm{r.Y4}$ & $1.4 . \leqslant 4$ & $1 \ldots .99$ & \multicolumn{2}{|c|}{ منتوسط الرش الورقي C } \\
\hline A.B & $\mathbf{C}$ & \multicolumn{2}{|c|}{ A.B.C } & \multirow{2}{*}{\multicolumn{2}{|c|}{ L. S. D 0.05}} \\
\hline 1.7 .7 & 1.117 & \multicolumn{2}{|c|}{ r.VAr } & & \\
\hline متوسط A & $\mathbf{C}_{2}$ & $\mathbf{C}_{1}$ & $\mathrm{C}_{0}$ & \multicolumn{2}{|c|}{ الأصل والرش الورقيA.C } \\
\hline 1.1 .00 & $1.0 .1 \varepsilon$ & $1 \ldots v r$ & 91.1. & \multicolumn{2}{|c|}{$\mathbf{A}_{1}$} \\
\hline 111.07 & 111.71 & $11 Y .14$ & $1 . r . \wedge 9$ & \multicolumn{2}{|c|}{$\mathbf{A}_{2}$} \\
\hline $.9 \mathrm{rV}$ & \multicolumn{3}{|c|}{1.7 .7} & \multicolumn{2}{|c|}{ L. S. D 0.05} \\
\hline متوسط B & $\mathbf{C}_{2}$ & $\mathrm{C}_{1}$ & $\mathrm{C}_{0}$ & \multicolumn{2}{|c|}{ التسميد الأرضي والرش الورقي B.C } \\
\hline $91 . .1$ & $97.9 \mathrm{~V}$ & 9Y.r4 & Ar.qr & \multicolumn{2}{|c|}{$\mathbf{B}_{0}$} \\
\hline 1.7 .81 & $111.1 \%$ & $1 \cdot 0 . \leqslant V$ & $1 . T .0 \leq$ & \multicolumn{2}{|c|}{$\mathbf{B}_{1}$} \\
\hline $1 \times 1 . \wedge 1$ & $1 Y 1.79$ & 111.54 & 110.01 & \multicolumn{2}{|c|}{$\mathbf{B}_{2}$} \\
\hline $1.1 \% 4$ & \multicolumn{3}{|c|}{$1.97 \mathrm{~V}$} & \multicolumn{2}{|c|}{ L .S. D 0.05} \\
\hline
\end{tabular}

ولوحظ وجود فروق معنوية لمعاملة الرش الورقي لصفة ارتفاع النبات إذ تفوق التركيز ·r مل.لنز - معنويا بإعطائها أعلى متوسط بلغ

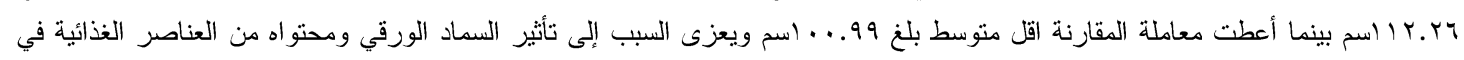

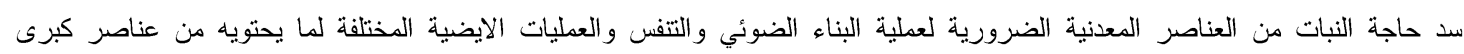

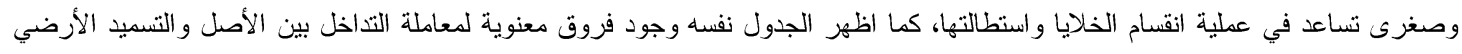


إذ تفوقت جميع المعاملات على معاملة المقارنة وأعطت المعاملة ...امل. لتر -سماد ارضي مع أصل الليمون الحلو أعلى متوسط

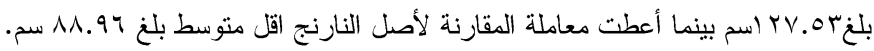

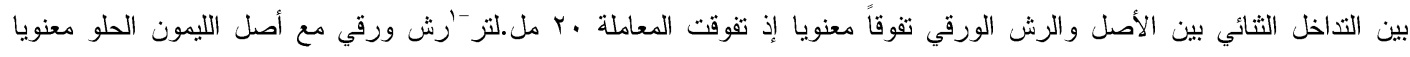

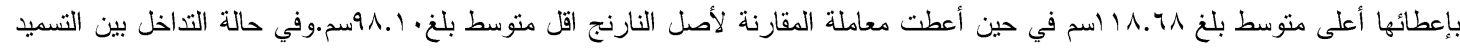

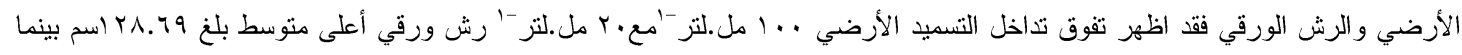

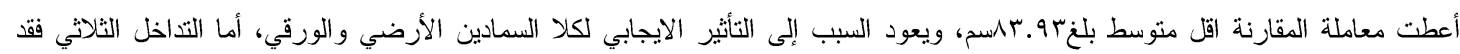

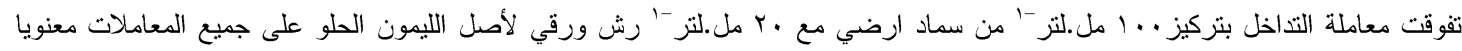

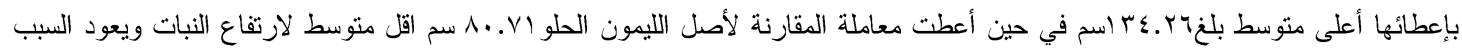
إلى التداخل بين عوامل التجربة.

أظهر جدول إن للأصل نأثير ا معنويا في متوسط قطر الأصل إذ تفوق أصل النارنج على أصل الليمون الحلو بإعطائه أعلى متوسط لقطر

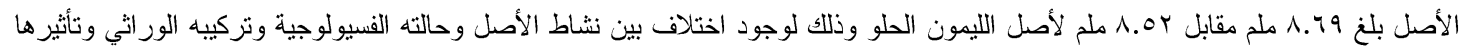

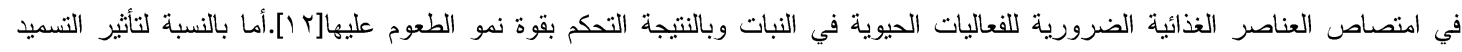

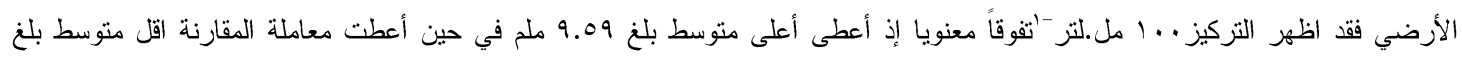

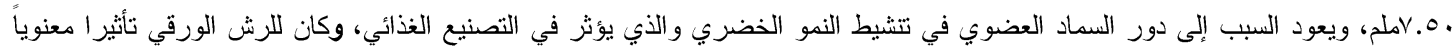

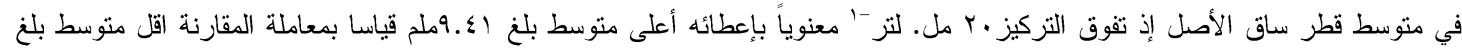

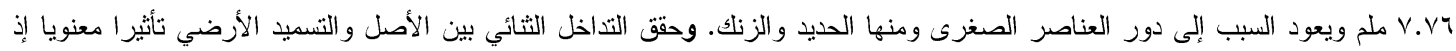

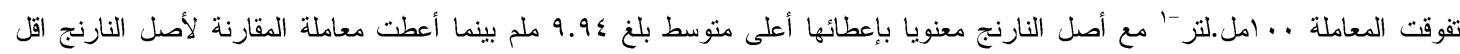

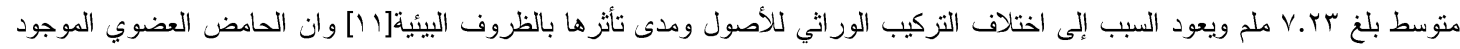

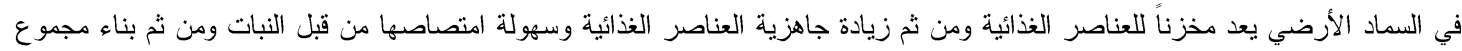

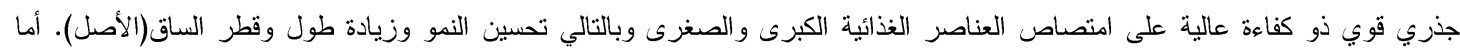

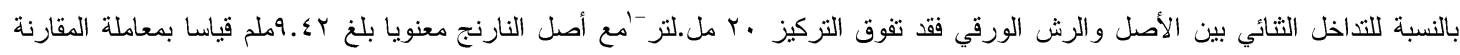

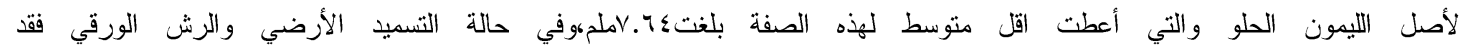

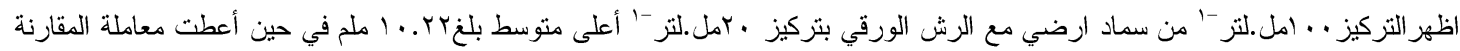

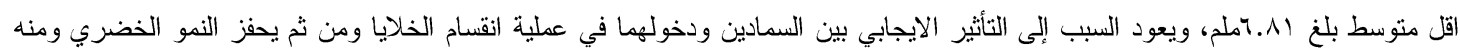

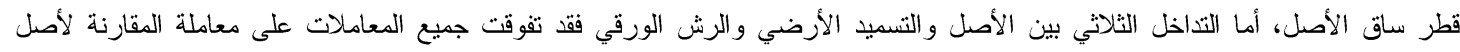

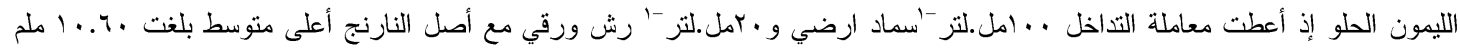

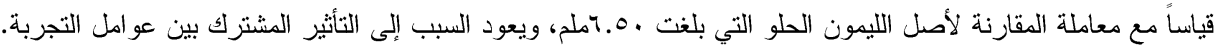

جدول(ך): تأثير الأصل و التغذية الأرضية والورقية في متوسط قطر الأصل (ملم) لثتلات الليمون حامض المحلي.

\begin{tabular}{|c|c|c|c|c|c|}
\hline \multirow[t]{2}{*}{ A.B } & \multicolumn{3}{|c|}{ 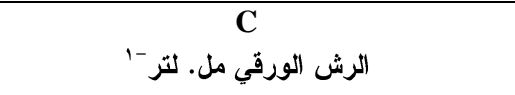 } & \multirow{2}{*}{ 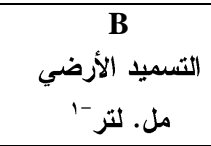 } & \multirow[t]{2}{*}{ الأصل } \\
\hline & $\mathbf{C}_{2}$ & $\mathbf{C}_{1}$ & $\mathrm{C}_{0}$ & & \\
\hline V.rr & V.rr & V.rT & V.IT & $\mathbf{B}_{\mathbf{0}}$ & \multirow[t]{3}{*}{ A1 النارنج A } \\
\hline$\Lambda .9 \mathrm{r}$ & $1 . . r \varepsilon$ & ᄉ.. $\mathrm{V}$ & V.VT & $\mathbf{B}_{1}$ & \\
\hline $9.9 \varepsilon$ & $1 . .7$ & $1 . . \leqslant V$ & A.vo & $\mathbf{B}_{2}$ & \\
\hline V.VA & 9.10 & V.VI & 7.0. & $\mathbf{B}_{0}$ & \multirow{3}{*}{ اللخو A2 } \\
\hline A.Or & 9.10 & $\wedge .0 \leqslant$ & V.Ar & $\mathbf{B}_{1}$ & \\
\hline \multirow[t]{2}{*}{ q.ro } & $9 . \wedge \varepsilon$ & 9.41 & 1. . & $\mathbf{B}_{2}$ & \\
\hline & $9 . \leqslant 1$ & 1.70 & V.VY & \multicolumn{2}{|c|}{ متوسط الرش الورقي C } \\
\hline A.B & $\mathbf{C}$ & \multicolumn{2}{|c|}{ A.B.C } & \multirow{2}{*}{\multicolumn{2}{|c|}{ L. S. D 0.05}} \\
\hline$\cdots 79$ & $\cdots \leqslant q$ & \multicolumn{2}{|c|}{. .119} & & \\
\hline متوسطA & $\mathbf{C}_{2}$ & $\mathrm{C}_{1}$ & $\mathrm{C}_{0}$ & \multicolumn{2}{|c|}{ الأصل والرش الورقيA.C } \\
\hline 1.79 & $9 . \leqslant Y$ & $\wedge . \vee q$ & $\vee . \wedge \Lambda$ & \multicolumn{2}{|c|}{$\overline{A_{1}}$} \\
\hline A.०r & 9.\&) & A.or & V.T & \multicolumn{2}{|c|}{$\mathbf{A}_{2}$} \\
\hline$\cdots \varepsilon$. & \multicolumn{3}{|c|}{$\cdots 79$} & \multicolumn{2}{|c|}{ L S D 0.05} \\
\hline متوسط B م & $\mathbf{C}_{2}$ & $\mathbf{C}_{1}$ & $\mathrm{C}_{0}$ & \multicolumn{2}{|c|}{ التسميد الأرضي والرش الورقي B.C } \\
\hline
\end{tabular}




\begin{tabular}{|c|c|c|c|c|}
\hline V.०. & $\Lambda . Y \leq$ & $V . \varepsilon V$ & 7.11 & $\mathbf{B}_{0}$ \\
\hline A.Vr & 9.19 & 1.. & V.Vq & $\mathbf{B}_{1}$ \\
\hline 9.09 & $1 . . r Y$ & $9 . \wedge 9$ & A.TV & $\mathbf{B}_{2}$ \\
\hline$\ldots \leqslant q$ & &.$\wedge \varepsilon$ & & L. S. D 0.05 \\
\hline
\end{tabular}

يظهز من جدول وجود فروق معنوية في صفة قطر الطعم إذ تفوق أصل النارنج معنويا على أصل الليمون الحلو بتحقيقه أعلى متوسط بلغ 7.84ملم مقارنه بأصل الليمون الحلو الذي بلغ 7.64ملم ويعود السبب إلى طول فترة النمو وملائمة الظروف البيائية لتكوين منطقه التحام جيدة

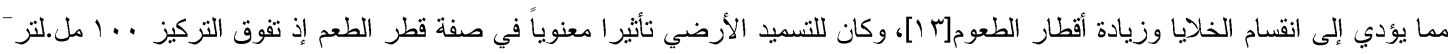

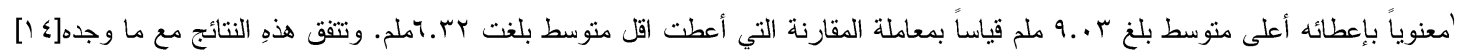

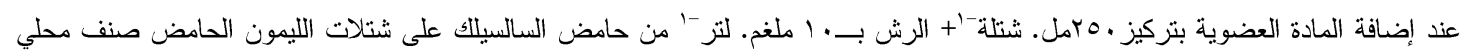

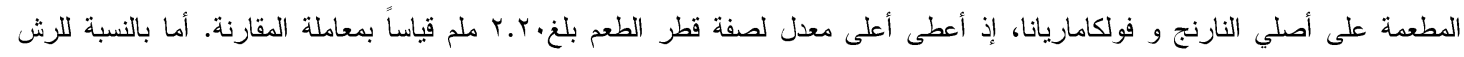

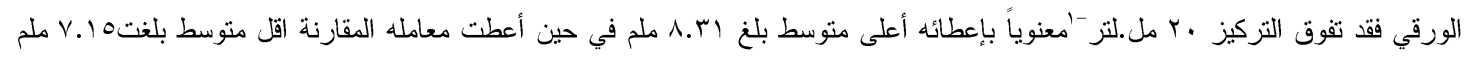

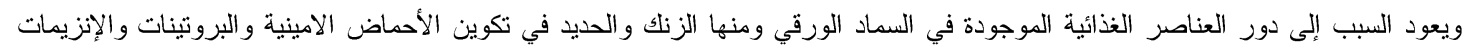

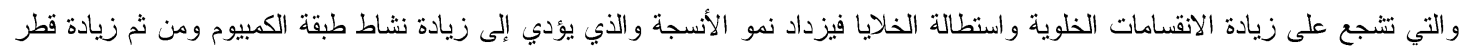

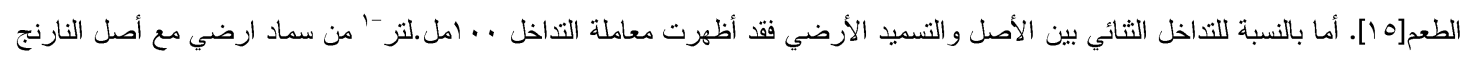

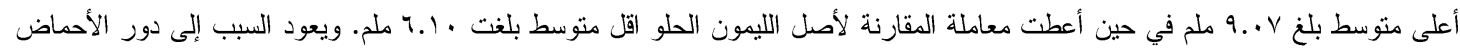

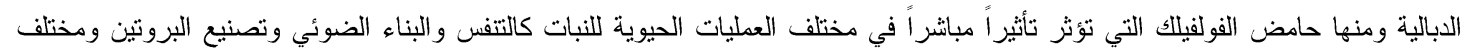

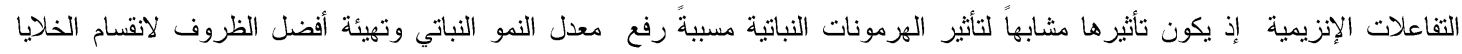

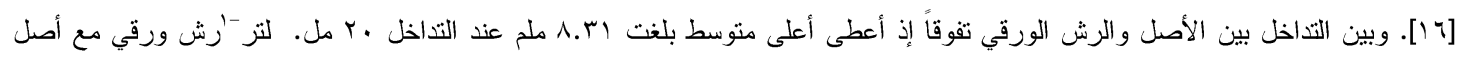

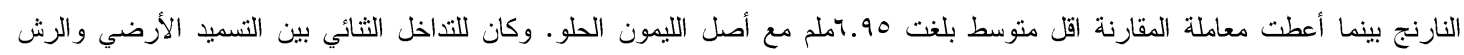

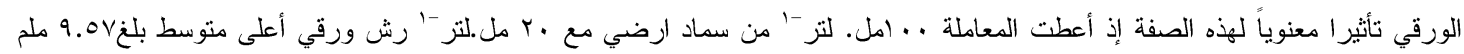

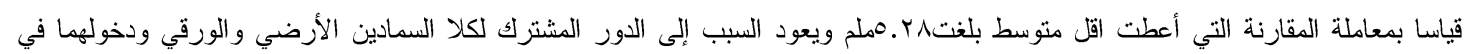

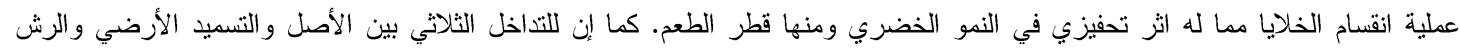

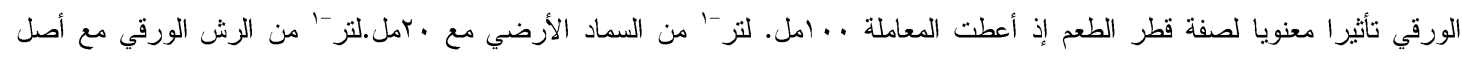

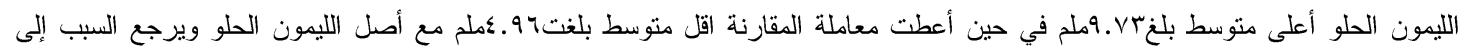

جدول(V): تأثير الأصل و التغذية الأرضية و الورقية في متوسط ثطر الطعم (ملم) لشتلات الليمون حامض المحلي.

\begin{tabular}{|c|c|c|c|c|c|}
\hline \multirow[t]{2}{*}{ A.B } & \multicolumn{3}{|c|}{ الرش الورقي مل. لتر - ' } & \multirow{2}{*}{ 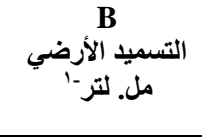 } & \multirow[t]{2}{*}{ الأصل } \\
\hline & $\mathbf{C}_{2}$ & $\mathbf{C}_{1}$ & $\mathrm{C}_{0}$ & & \\
\hline 7.00 & $\vee . r \wedge$ & 7.74 & 0.71 & $\mathbf{B}_{0}$ & \multirow[t]{3}{*}{ النارنج A1 } \\
\hline$V .9 Y$ & $\Lambda .10$ & $V .9 Y$ & 8.79 & $\mathbf{B}_{1}$ & \\
\hline $9 . \cdot v$ & $9 . Y \leq$ & $9 . .0$ & $\Lambda . \vee \theta$ & $\mathbf{B}_{2}$ & \\
\hline 7.1. & $7 . r V$ & 7.74 & $\$ .97$ & $\mathbf{B}_{\mathbf{0}}$ & \multirow{3}{*}{ الليمون الحلو } \\
\hline$\vee . \wedge r$ & $\Lambda . \leqslant 4$ & V.VI & V.rr & $\mathbf{B}_{1}$ & \\
\hline \multirow[t]{2}{*}{1.99} & $9 . \vee r$ & $\Lambda .79$ & $\Lambda .0 \Lambda$ & $\mathbf{B}_{2}$ & \\
\hline & $\Lambda . M I$ & V.VV & 8.10 & \multicolumn{2}{|c|}{ متوسط الرش الورقي C } \\
\hline A.B & $\mathbf{C}$ & \multicolumn{2}{|c|}{ A.B.C } & \multirow{2}{*}{\multicolumn{2}{|c|}{ L. S. D 0.05}} \\
\hline. $.17 \mathrm{~V}$ &. .111 & \multicolumn{2}{|c|}{$\because r \wedge q$} & & \\
\hline متوسط A & $\mathrm{C}_{2}$ & $\mathrm{C}_{1}$ & $\mathrm{C}_{0}$ & \multicolumn{2}{|c|}{ الأصل والرش الورقيA.C } \\
\hline$\vee . \wedge \varepsilon$ & $\Lambda . \mu 1$ & $\mathrm{v} . \wedge \mathrm{V}$ & V.ro & \multicolumn{2}{|c|}{$\overline{A_{1}}$} \\
\hline V.T\& & A.r. & $\mathrm{V} . \mathrm{TV}$ & 7.90 & \multicolumn{2}{|c|}{$\mathbf{A}_{2}$} \\
\hline$\because .97$ & \multicolumn{3}{|c|}{$.17 \mathrm{~V}$} & \multicolumn{2}{|c|}{ L. S. D 0.05} \\
\hline Bتوسط B & $\mathbf{C}_{2}$ & $\mathrm{C}_{1}$ & $\mathrm{C}_{0}$ & \multicolumn{2}{|c|}{ التسميد الأرضي والرش الورقي B.C } \\
\hline $7 . r 4$ & $v_{.0} 0$ & $7.7 \varepsilon$ & $0 . Y \Lambda$ & \multicolumn{2}{|c|}{$\mathbf{B}_{0}$} \\
\hline$\vee . \wedge \vee$ & ^.r. & V.AI & 0.01 & \multicolumn{2}{|c|}{$\mathbf{B}_{1}$} \\
\hline $9 . . r$ & $9.0 \mathrm{~V}$ & $\Lambda . \wedge \mathrm{V}$ & 1.74 & \multicolumn{2}{|c|}{$\mathbf{B}_{2}$} \\
\hline .111 & \multicolumn{3}{|c|}{.$r \cdot \theta$} & \multicolumn{2}{|c|}{ L. S. D 0.05 } \\
\hline
\end{tabular}


يوضح جدولى إن للأصل تأثيراً معنوياً في متوسط عدد الأوراق إذ تقّوق أصل الليمون الحلو معنوياً وأعطى أعلى منوسط لصفة عدد

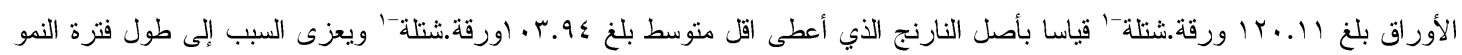

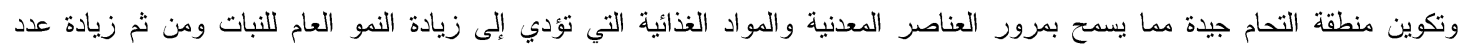

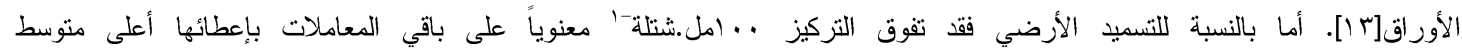

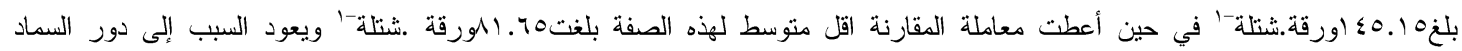

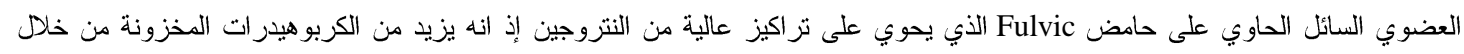

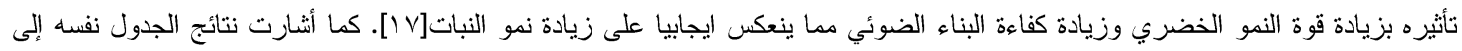

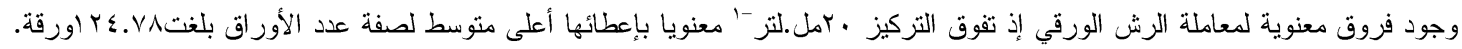

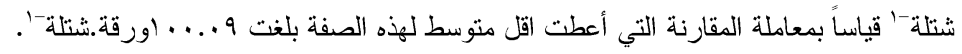

جدول(^): تأثير الأصل و التغذية الأرضية والورقية في متوسط عدد الأوراق (ورقة. شتثة-1') لشتلات الليمون حامض المحلي .

\begin{tabular}{|c|c|c|c|c|c|}
\hline \multirow[t]{2}{*}{ A.B } & \multicolumn{3}{|c|}{ 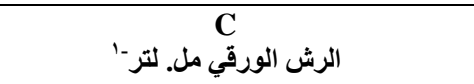 } & \multirow[t]{2}{*}{ التسميد الأرضي مل.لتز -' } & \multirow[t]{2}{*}{ الأصول } \\
\hline & $\mathrm{C}_{2}$ & $\mathrm{C}_{1}$ & $\mathrm{C}_{0}$ & & \\
\hline Vr.91 & AV.rY & $\vee 9.59$ & 00.11 & $\overline{\mathbf{B}_{0}}$ & \multirow[t]{3}{*}{ النارنج A1 } \\
\hline $1 \cdot \leq .0 \leq$ & $11 r . \wedge 9$ & 1.r.rr & 99.4 & $\mathbf{B}_{1}$ & \\
\hline Irr.r. & 104.74 & $1 \times 4.07$ & $M \cdot . V T$ & $\mathbf{B}_{2}$ & \\
\hline AQ.rY & $9 V . r 4$ & 91.24 & Vq.r. & $\mathbf{B}_{0}$ & \multirow{3}{*}{$\begin{array}{c}\text { الليمون الحلو } \\
\text { A2 }\end{array}$} \\
\hline $11 \leq . \cdot 1$ & 119.14 & 110.17 & $1 \cdot v .91$ & $\mathbf{B}_{1}$ & \\
\hline 107.99 & IVV.T4 & $101 . \mathrm{rr}$ & $1 \leqslant Y .$. & $\mathbf{B}_{2}$ & \\
\hline & $T r \xi . V A$ & $111 . r 1$ & $1 \cdots .9$ & \multicolumn{2}{|c|}{ متوسط الرش الورقي } \\
\hline A.B & $\mathbf{C}$ & \multicolumn{2}{|c|}{$\begin{array}{c}\text { A. B.C } \\
\text { التداخل الثلاثي }\end{array}$} & \multirow{2}{*}{\multicolumn{2}{|c|}{ L. S. D 0.05}} \\
\hline$r .790$ & 1.9 .0 & \multicolumn{2}{|c|}{$\varepsilon .74 \mathrm{~V}$} & & \\
\hline متوسط A & $\mathbf{C}_{2}$ & $\mathrm{C}_{1}$ & $\mathbf{C}_{0}$ & \multicolumn{2}{|c|}{ الأصل والرش الورقي_A.C } \\
\hline $1 \cdot 4.9 \leq$ & 118.90 & $1 . r .11$ & $9 \cdot . \mathrm{VV}$ & \multicolumn{2}{|l|}{$\overline{A_{1}}$} \\
\hline $14 \cdot .11$ & 111.71 & 119.4. & $1.9 . \leqslant Y$ & \multicolumn{2}{|l|}{$\mathbf{A}_{2}$} \\
\hline 1.007 & \multicolumn{3}{|c|}{9.790} & \multicolumn{2}{|c|}{ L. S. D 0.05} \\
\hline B متوسط B & $\mathbf{C}_{2}$ & $\mathbf{C}_{1}$ & $\mathrm{C}_{0}$ & \multicolumn{2}{|c|}{ التسميد الأرضي والرش الورقي B.C } \\
\hline 11.70 & QY.rY & 10. $\leqslant \leqslant$ & $7 V .19$ & \multicolumn{2}{|c|}{$\mathbf{B}_{0}$} \\
\hline $1.9 . \Lambda$ & $117 . \wedge \mathrm{V}$ & $1.9 .5 \leq$ & $1.1 . V 4$ & \multicolumn{2}{|l|}{$\mathbf{B}_{1}$} \\
\hline $1 \leq 0.10$ & 170.17 & $1 \% \wedge .9 \leq$ & 151.54 & \multirow{2}{*}{\multicolumn{2}{|c|}{$\begin{array}{c}\text { B }_{2} \\
\text { L. S. D } 0.05\end{array}$}} \\
\hline 1.9 .0 & \multicolumn{3}{|c|}{ r.r... } & & \\
\hline
\end{tabular}

ويعود السبب إلى دور العناصر الغذائية الموجودة في السماد الورقي وتحفيزها للقيام بعلية البناء الضوئي والتتفس والعمليات الايضية الأخرى

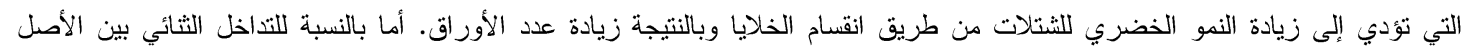

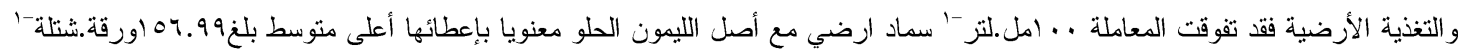

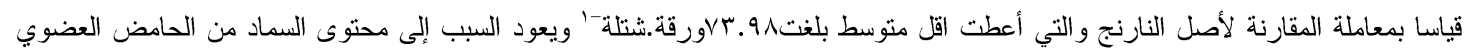

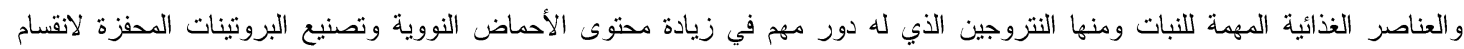

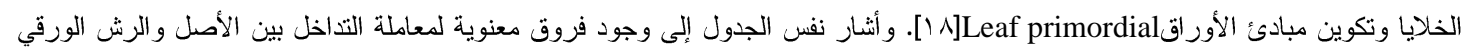

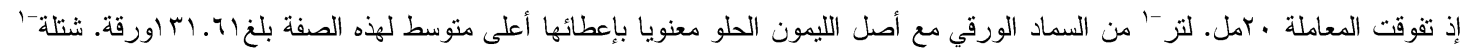

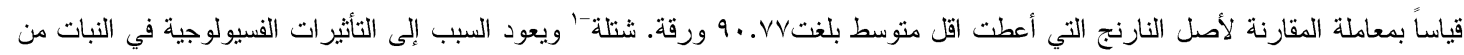

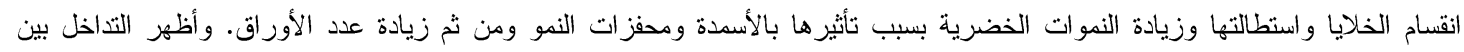

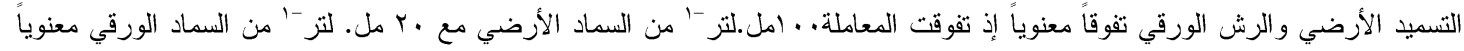

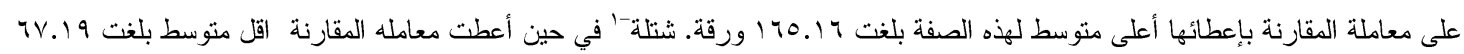

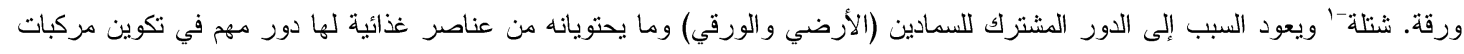

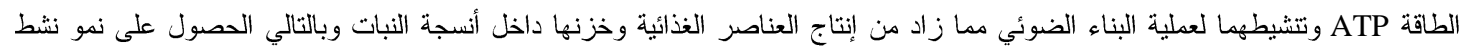

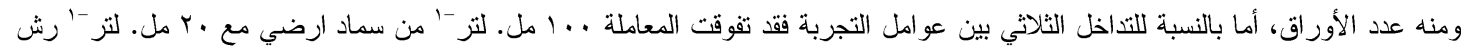

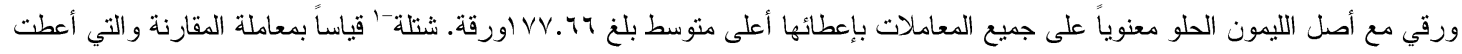




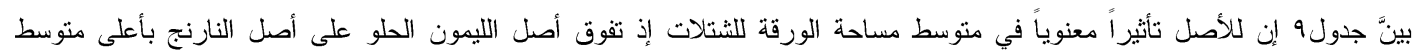

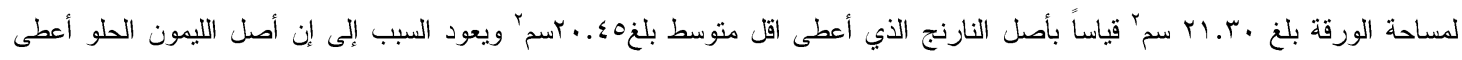

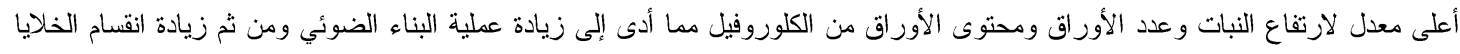

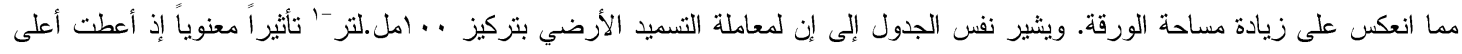

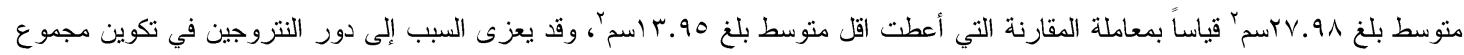

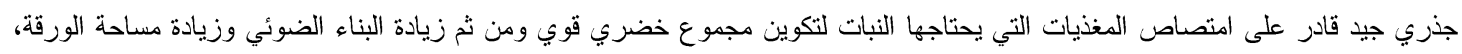

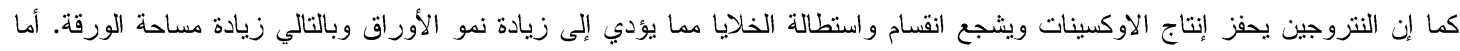

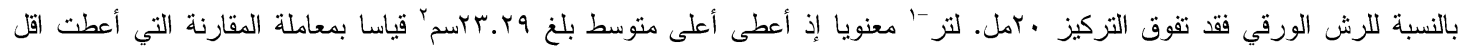

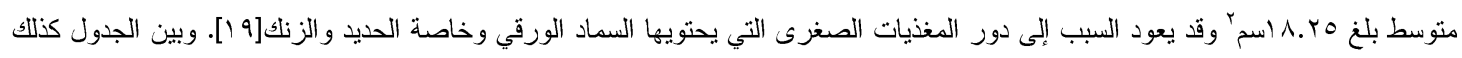

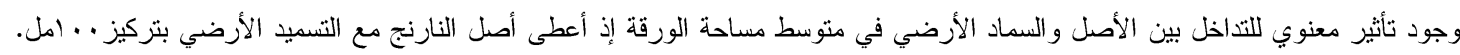

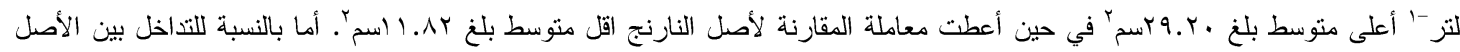

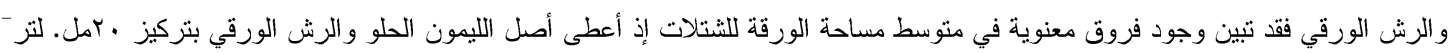

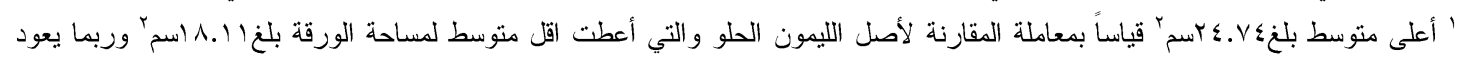

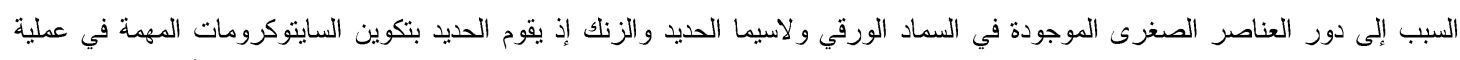

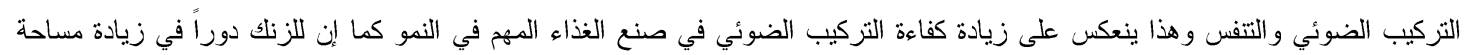

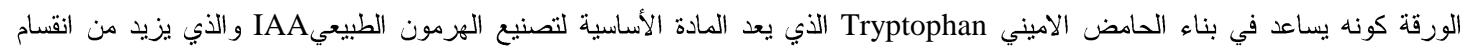

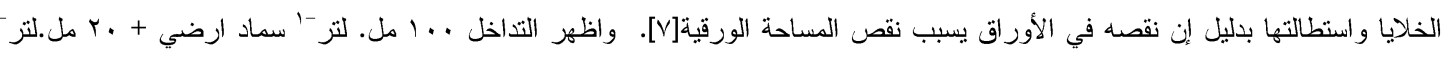

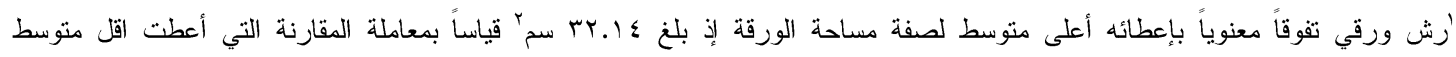

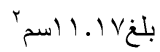

ويعود السبب إلى الدور المشترك للتغذية الأرضية والتخذية الورقية وما تحويه من عناصر كبرى وصغرى ودخولهما في عملية انقسام الخلايا

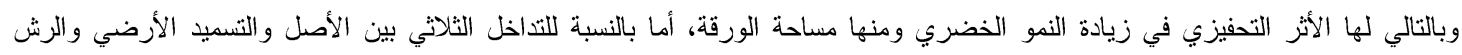

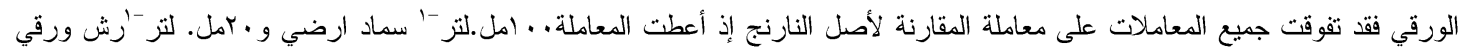

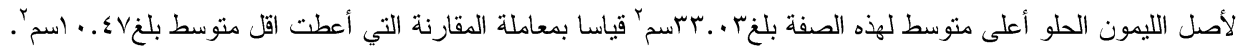

جدول(9): تأثير الأصل و التغذية الأرضية و الورقية في متوسط مساحة الورقة (سمَ) لشتلات الليمون حامض المحلي.

\begin{tabular}{|c|c|c|c|c|c|}
\hline \multirow[t]{2}{*}{ A.B } & \multicolumn{3}{|c|}{ 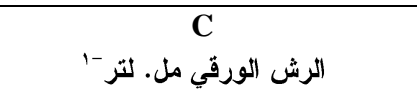 } & \multirow{2}{*}{ 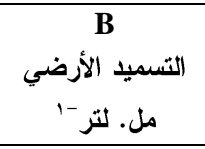 } & \multirow[t]{2}{*}{$\begin{array}{l}\text { A } \\
\end{array}$} \\
\hline & $\mathrm{C}_{2}$ & $\mathrm{C}_{1}$ & $\mathrm{C}_{0}$ & & \\
\hline $11 . \wedge r$ & IT.YA & $11 . V r$ & $1 . . \leqslant V$ & $\mathbf{B}_{0}$ & \multirow[t]{3}{*}{ A1 النارنج } \\
\hline$r . . r \leq$ & r1... & Yl.AT & 11.17 & $\mathbf{B}_{1}$ & \\
\hline rq.Y. & MI.Y & ra.A. & YY.0T & $\mathbf{B}_{2}$ & \\
\hline $17 . .1$ & $19 . \leqslant V$ & 17.9. & $11 . \wedge \mathrm{V}$ & $\mathbf{B}_{0}$ & \multirow{3}{*}{$\begin{array}{c}\text { الليمون الحلو } \\
\text { A2 }\end{array}$} \\
\hline r1... & rI.Vr & $r 1.1 r$ & $r \cdot r \cdot r$ & $\mathbf{B}_{1}$ & \\
\hline \multirow[t]{2}{*}{ rT.VV } & rr..r & ro.1r & TY.19 & $\mathbf{B}_{2}$ & \\
\hline & rr.rq & $r 1 . .9$ & IA.ro & \multicolumn{2}{|c|}{ متوسط الرش الورقي C } \\
\hline A.B & $\mathbf{C}$ & \multicolumn{2}{|c|}{ A.B.C } & \multirow{2}{*}{\multicolumn{2}{|c|}{ L. S. D 0.05}} \\
\hline. .7 .1 &..$\varepsilon r$ & \multicolumn{2}{|c|}{1.00} & & \\
\hline A مثوسط A & $\mathrm{C}_{2}$ & $\mathrm{C}_{1}$ & $\mathrm{C}_{0}$ & \multicolumn{2}{|c|}{ الأصل والرش الورقيA.C } \\
\hline r...so & $Y 1 . \wedge \varepsilon$ & $r 1.1 r$ & $11 . r 9$ & \multicolumn{2}{|c|}{$\mathbf{A}_{1}$} \\
\hline rI.r. & $r \varepsilon . V \leqslant$ & r1..0 & 11.11 & \multicolumn{2}{|c|}{$\mathbf{A}_{2}$} \\
\hline..$r 01$ & \multicolumn{3}{|c|}{.7 .1} & \multicolumn{2}{|c|}{ L. S. D 0.05} \\
\hline B منوسط & $\mathrm{C}_{2}$ & $\mathrm{C}_{1}$ & $\mathrm{C}_{0}$ & \multicolumn{2}{|c|}{ التسميد الأرضي والرش الورقي B.C } \\
\hline 14.90 & 19.rV & IE.T1 & $11.1 \mathrm{~V}$ & \multicolumn{2}{|c|}{$\mathbf{B}_{0}$} \\
\hline$r . .79$ & rl.rt & r1. $\{9$ & 19.rr & \multicolumn{2}{|c|}{$\mathbf{B}_{1}$} \\
\hline$r V .9 \Lambda$ & Mr.ls & YV.\&T & Y\&.MT & \multicolumn{2}{|c|}{$\mathbf{B}_{2}$} \\
\hline 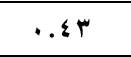 & \multicolumn{3}{|c|}{$. V \leqslant 0$} & \multicolumn{2}{|c|}{ L. S. D 0.05} \\
\hline
\end{tabular}




\section{CONFLICT OF INTERESTS}

There are no conflicts of interest.

[1] الخفاجي، مكي علوان، سهيل عليوي عطرة وعلاء عبد الرزاق. الفاكهة المستديمة الخضرة. وزارة التعليم العالي والبحث العلمي. جامعة

بغداد. العراق. . 199. 199.

[ب] الجريان، أسر اء لؤي حمدان. فاكهة اللالنكي يوسف أفندي. وزارة التعليم العالي والبحث العلمي. جامعة بــــــلـ كلية الزراعة ـ العراق.

$$
. r \cdot 11
$$

[ب] الجميلي، علاء عبد الرزاق وجبار عباس حسن الدجيلي. إنتاج الفاكهة.وزارة التعليم العالي والبحث العلمي. جامعة بغداد. العراق. 1919. [₹] Fort, C. A. Health Beneficial Citrus Compounds. Senior Thesis, Bachelor of Arts. Bard College at Simons Rock Garrington , Massachusetts.71 p. 2011.

[0] سعد الله. محمد حسين ومحمد سامي المليجي. زراعة الموالح. مطابع الدعم الإعلامي بالاسماعيلية، معهد كوت البساتين، مركز البحوث

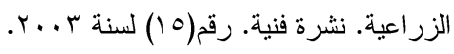

[7] Abd- Elmohsen, M.EL- Bassiony. 2003. Response of some bean (Phaseolusvugaris L.) cultivars to organic and Bio fertilizer Ph. D. Thesis, Fac. Agric. Ain Shams University.

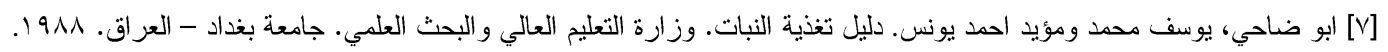

[^]Black, C.A.Ed. Methods of Soil Analysis. Part 2.Amer.Soc. Agro. Madison, Wisconson.USA. 1965.

$$
\begin{aligned}
& \text { [9] الصحاف، فاضل حسين(a). أنظمة الزراعة بدون استخدام تربة. بيت الحكمة- جامعة بغدادوزارة التعلم العالي والبحث العلمي- جمهورية }
\end{aligned}
$$

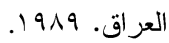

$$
\begin{aligned}
& \text { [• ا]لالر اوي، خانتع محمود وعبد العزيز محمد خلف الله. تصميم وتحليل التجارب الزراعية. وزارة التعليم العالي والبحث العلمي. العراق . }
\end{aligned}
$$

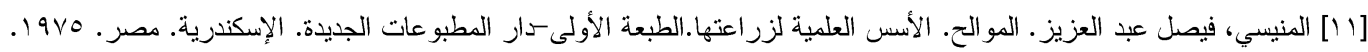

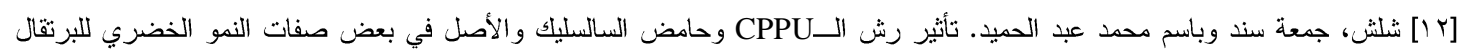

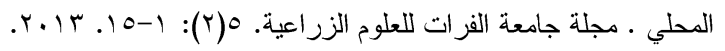

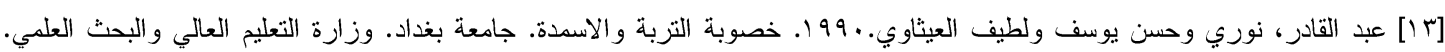

$$
\begin{aligned}
& \text { العراق. } \\
& \text { [؛ 1] العباسي، غالب بهيو عبود و علاء عيدان حسن و مسلم عبد علي الحسين. استجابة ثلاثة أصول من الحضيات للتسميد العضوي و الصخر }
\end{aligned}
$$

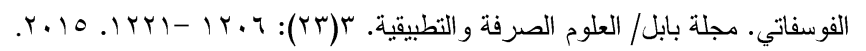

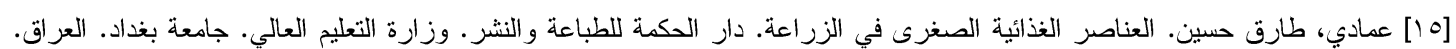

[17] Kulikova, N.A.; Dashitsyr enova, A.D.; Perminova, I.V. and Lebedeva, G.F. Auxin-like activity of different fractions of coal humic acids. Bulgarian J. Ecol. Sci., 2(3-4): 55-56 . 2003.

$[\mathrm{V}$ ] Keller, M. and M. Koblet. Dry matter and leaf area partitioning bud fertility and second season growth Vitisvinifera L. : Response to nitrogen supply and limiting irradiance. Vitis. 34 (2) : 77-83. 1995.

[1 1 ] Coartney. J. S., More. D. J. and key J. L.1967.Inhibition of RNA synthesis and auxin-induced cell wall extensibility and growth by actinomycin. Plant physiol. 42:434-437.

$$
\text { [9 19 الصحاف, فاضل حسين. تغذية النبات التطبيقي. وزارة التعليم العالي و البحث العلمي.جامعة بغداد بيت الحكمة. } 9199 .
$$

